\title{
Noninvasive Biomarkers Identify Eosinophilic Esophagitis: A Prospective Longitudinal Study in Children
}

\author{
Joshua Wechsler ${ }^{1}$, Steven Ackerman ${ }^{2}$, Mirna Chehade ${ }^{3}$, Katie Amsden ${ }^{1}$, Mary Ellen Riffle ${ }^{3}$, \\ Ming-Yu Wang ${ }^{1}$, Jian Du², Matthew Kleinjan ${ }^{2}$, Preeth Alumkal ${ }^{2}$, Elizabeth Gray ${ }^{4}$, \\ Kwang-Youn Kim ${ }^{4}$, Barry Wershil ${ }^{1}$, and Amir Kagalwalla ${ }^{1}$ \\ ${ }^{1}$ Ann \& Robert H. Lurie Children's Hospital \\ ${ }^{2}$ University of Illinois at Chicago \\ ${ }^{3}$ Mount Sinai School of Medicine \\ ${ }^{4}$ Northwestern University Feinberg School of Medicine
}

October 26, 2020

\begin{abstract}
Background: Esophageal histology is critical for diagnosis and surveillance of disease activity in eosinophilic esophagitis (EoE). A validated noninvasive biomarker has not been identified. We aimed to determine the utility of blood and urine eosinophilassociated proteins to identify EoE diagnosis and predict esophageal eosinophilia. Methods: Blood and urine were collected from children undergoing endoscopy with biopsy. Absolute eosinophil count (AEC), plasma eosinophil-derived neurotoxin (EDN), eosinophil cationic protein (ECP), major basic protein-1 (MBP-1), galectin-10 (CLC/GAL-10), Eotaxin-2 and Eotaxin-3, and urine osteopontin (OPN) and matrix metalloproteinase-9 (MMP-9) were determined. Differences were assessed between EoE and control, and with treatment response. The capacity to predict EoE diagnosis and esophageal eosinophil counts was assessed. Results: 183 specimens were collected from 56 EoE patients and 15 non-EoE patient controls; 33 EoE patients had paired preand post-treatment specimens. Plasma (CLC/GAL-10, ECP, EDN, Eotaxin-3, MBP-1) and urine (OPN) biomarkers were increased in EoE compared to control. A panel comprising CLC/GAL-10, Eotaxin-3, ECP, EDN, MBP-1, and AEC was superior to AEC alone in distinguishing EoE from control. AEC, CLC/GAL-10, ECP, and MBP-1 were significantly decreased in patients with a good response to treatment compared to patients with a poor response. AEC, CLC/GAL-10, ECP, EDN, OPN, and MBP-1 each predicted esophageal eosinophil counts utilizing mixed models controlled for age, gender, treatment and atopy; AEC combined with MBP-1 best predicted the counts. Conclusions: We identified novel panels of eosinophil-associated proteins that along with AEC are superior to AEC alone in distinguishing EoE from control and predicting esophageal eosinophil counts.
\end{abstract}

\section{Hosted file}

Non-invasive biomarker manuscript figures tables.pdf available at https://authorea.com/ users/370157/articles/488886-noninvasive-biomarkers-identify-eosinophilic-esophagitis-aprospective-longitudinal-study-in-children 